TRANSACTIONS OF THE

AMERICAN MATHEMATICAL SOCIETY

Volume 351, Number 12, Pages 4963-4980

S 0002-9947(99)02241-2

Article electronically published on August 27, 1999

\title{
LOWER BOUNDS FOR THE ABSOLUTE VALUE OF RANDOM POLYNOMIALS ON A NEIGHBORHOOD OF THE UNIT CIRCLE
}

\author{
S. V. KONYAGIN AND W. SCHLAG
}

\begin{abstract}
Let $T(x)=\sum_{j=0}^{n-1} \pm e^{i j x}$ where \pm stands for a random choice of sign with equal probability. The first author recently showed that for any $\epsilon>0$ and most choices of sign, $\min _{x \in[0,2 \pi)}|T(x)|<n^{-1 / 2+\epsilon}$, provided $n$ is large. In this paper we show that the power $n^{-1 / 2}$ is optimal. More precisely, for sufficiently small $\epsilon>0$ and large $n$ most choices of sign satisfy $\min _{x \in[0,2 \pi)}|T(x)|>\epsilon n^{-1 / 2}$. Furthermore, we study the case of more general random coefficients and applications of our methods to complex zeros of random polynomials.
\end{abstract}

\section{INTRODUCTION}

Let $r_{0}, r_{1}, \ldots$ be Rademacher variables, i.e., they are i.i.d. with $\mathbb{P}\left(\left\{r_{0}=1\right\}\right)=$ $\mathbb{P}\left(\left\{r_{0}=-1\right\}\right)=\frac{1}{2}$. We define

$$
P_{n}(u)=\mathbb{P}\left(\left\{\min _{x \in \mathbb{T}}\left|\sum_{j=0}^{n-1} r_{j} e^{i j x}\right|>u\right\}\right)
$$

for $u \geq 0$. As usual, $\mathbb{T}=\mathbb{R} / 2 \pi \mathbb{Z}$. Note that $P_{n}(u)$ is non-increasing and that $P_{n}(\sqrt{n})=0$. Littlewood [7] conjectured that $P_{n}(\epsilon \sqrt{n}) \rightarrow 0$ as $n \rightarrow \infty$ for any $\epsilon>0$. This was proved by Kashin [5], who showed that $\lim _{n \rightarrow \infty} P_{n}\left(n^{\frac{1}{2}}(\log n)^{-\frac{1}{3}}\right)=0$. In [6] the first author proved a conjecture of Odlyzko, namely $\lim _{n \rightarrow \infty} P_{n}\left(n^{-\frac{1}{2}+\epsilon}\right)=$ 0 for any $\epsilon>0$. It is easy to see that the method from [6] applies not only to $T=\sum_{j=0}^{n-1} r_{j} e^{i j x}$ but also to $T^{\prime}, T^{\prime \prime}$ etc. More precisely, with some obvious modifications the arguments from [6] yield the following:

Theorem 1.1. For any $\epsilon>0$ and any nonnegative integer $\nu$

$$
\lim _{n \rightarrow \infty} \mathbb{P}\left(\left\{\min _{x \in \mathbb{T}}\left|\sum_{j=0}^{n-1} r_{j}(j / n)^{\nu} e^{i j x}\right|>n^{-\frac{1}{2}+\epsilon}\right\}\right)=0 .
$$

Other extensions of the methods in [6] are in [4], where the case of i.i.d. $r_{j}$ satisfying suitable moment conditions is considered. In this paper we show that the power $n^{-\frac{1}{2}}$ is optimal. More precisely, we prove

Received by the editors February 5, 1997 and, in revised form, September 24, 1997.

1991 Mathematics Subject Classification. Primary 42A05, 42A61; Secondary 30C15, 60F05.

The authors were supported by the National Science Foundation, grant DMS 9304580. This research was carried out while the authors were members of the Institute for Advanced Study, Princeton. It is a pleasure to thank the Institute for its hospitality and generosity. The authors would like to thank A. G. Karapetian for comments on a preliminary version of this paper.

(C)1999 American Mathematical Society 
Theorem 1.2. Let $r_{0}, r_{1}, \ldots$ be standard normal or Rademacher variables and suppose that $\phi \in C^{\sigma}([0,1]) \backslash\{0\}$ for some $\sigma \in(1 / 2,1]$. Then for any $\epsilon>0$

$$
\limsup _{n \rightarrow \infty} \mathbb{P}\left(\left\{\min _{z \in \mathbb{C}:|| z|-1|<\epsilon n^{-2}}\left|\sum_{j=0}^{n-1} r_{j} \phi(j / n) z^{j}\right|<\epsilon n^{-\frac{1}{2}}\right\}\right) \leq C \epsilon .
$$

Here $C$ is a constant depending only on $\phi$.

$C^{\sigma}$ is the space of all real-valued Hölder continuous functions of order $\sigma$ on $[0,1]$. It is possible that Theorem 1.2 holds for less regular functions $\phi$, but our method seems to have $\sigma=1 / 2$ as a natural threshold.

In [8] Shepp and Vanderbei study random polynomials $p(z)=\sum_{j=0}^{n-1} r_{j} z^{j}$ with standard normal coefficients. They show that for large $n$ the zeros of $p$ will lie close to the unit circle or the real axis. Moreover, they conjecture that with high probability the polynomial $p$ vanishes at some point in a $O\left(n^{-2}\right)$-neighborhood of the unit circle. Theorem 1.2 shows that this conjecture is best possible. Real and complex zeros of random polynomials have been studied by various authors and we do not intend this paper as an introduction to the subject. The reader will find several references to the literature on random polynomials in [8].

\section{THE MAIN REDUCTION}

To motivate our proof of Theorem 1.2, we indicate how to obtain a weaker estimate for standard normal coefficients. For simplicity, we set $\phi=1$ and consider only minima over $|z|=1$. Let $n \geq 2, \gamma>\frac{1}{2}$ and choose non-overlapping intervals $I_{\alpha}$ such that

$$
\left\{x \in \mathbb{T}: n^{-\frac{11}{10}}<|x|<\pi-n^{-\frac{11}{10}}\right\} \subset \bigcup_{\alpha=1}^{N} I_{\alpha}
$$

with $(|\cdot|$ denotes the length of an interval $)$

$$
\left|I_{\alpha}\right| \leq n^{-2}(\log n)^{-\frac{1}{2}-\gamma}, N \leq 2 \pi n^{2}(\log n)^{\frac{1}{2}+\gamma} .
$$

For each $\alpha$ fix an $x_{\alpha} \in I_{\alpha}$ and let $T(x)=\sum_{j=0}^{n-1} r_{j} e^{i j x}$. Then, with some suitable absolute constant $C_{0}$ and for all large $n$,

$$
\begin{aligned}
& \mathbb{P}\left(\left\{\min _{x \in \mathbb{T}}|T(x)|<n^{-\frac{1}{2}}(\log n)^{-\gamma}\right\}\right) \\
\leq & \sum_{\alpha=1}^{N} \mathbb{P}\left(\left\{\min _{x \in I_{\alpha}}|T(x)|<n^{-\frac{1}{2}}(\log n)^{-\gamma},\left\|T^{\prime}\right\|_{\infty} \leq C_{0} n^{\frac{3}{2}}(\log n)^{\frac{1}{2}}\right\}\right) \\
& +2 \mathbb{P}\left(\left\{\min _{|x|<n^{-\frac{11}{10}}}|T(x)|<n^{-\frac{1}{2}}(\log n)^{-\gamma},\left\|T^{\prime}\right\|_{\infty} \leq C_{0} n^{\frac{3}{2}}(\log n)^{\frac{1}{2}}\right\}\right) \\
& +\mathbb{P}\left(\left\{\left\|T^{\prime}\right\|_{\infty}>C_{0} n^{\frac{3}{2}}(\log n)^{\frac{1}{2}}\right\}\right) \\
\leq & \sum_{\alpha=1}^{N} \mathbb{P}\left(\left\{\left|T\left(x_{\alpha}\right)\right|<2 C_{0} n^{-\frac{1}{2}}(\log n)^{-\gamma}\right\}\right) \\
& +2 \mathbb{P}\left(\left\{|T(0)|<n^{\frac{1}{2}-\frac{1}{20}}\right\}\right)+\mathbb{P}\left(\left\{\left\|T^{\prime}\right\|_{\infty}>C_{0} n^{\frac{3}{2}}(\log n)^{\frac{1}{2}}\right\}\right) .
\end{aligned}
$$

The factor 2 in (2.1) arises since $T(x)$ and $T(x+\pi)$ are identically distributed. To pass from (2.1) to (2.2), Taylor expand $T$ around $x_{\alpha}$ and zero to first order. Note 
that the last two terms are $o(1)$ by the DeMoivre-Laplace theorem and the SalemZygmund inequality, respectively (for the latter see [3], chapter 6, Theorem 2). Using characteristic functions, one checks that $\frac{1}{\sqrt{n}} T\left(x_{\alpha}\right)$ is again a Gaussian vector in $\mathbb{R}^{2}$ with mean zero and covariance matrix

$$
V_{\alpha}=\frac{1}{n} \sum_{j=0}^{n-1} \operatorname{cov}\left(r_{j}\left(\cos \left(j x_{\alpha}\right), \sin \left(j x_{\alpha}\right)\right)\right)=\frac{1}{n} \sum_{j=0}^{n-1}\left[\begin{array}{ll}
\cos ^{2}\left(j x_{\alpha}\right) & \frac{1}{2} \sin \left(2 j x_{\alpha}\right) \\
\frac{1}{2} \sin \left(2 j x_{\alpha}\right) & \sin ^{2}\left(j x_{\alpha}\right)
\end{array}\right] .
$$

A simple calculation (see Lemma 3.2) shows that $V_{\alpha}$ is uniformly nonsingular in $\alpha$ and sufficiently large $n$ provided $\frac{1}{n}<\left|x_{\alpha}\right|<\pi-\frac{1}{n}$, whereas the eigenvalues $\lambda_{\alpha}, \Lambda_{\alpha}$ of $V_{\alpha}$ satisfy

$$
C_{0}^{-1} \leq \Lambda_{\alpha} \leq C_{0} \quad, \lambda_{\alpha} \geq C_{0}^{-1} n^{-1 / 5}
$$

in the range

$$
\mathcal{B}=\left\{n^{-\frac{11}{10}}<\left|x_{\alpha}\right|<n^{-1}\right\} \cup\left\{\pi-n^{-1}<\left|x_{\alpha}\right|<\pi-n^{-\frac{11}{10}}\right\} .
$$

Thus the sum over $\alpha$ in (2.2) is bounded by a constant times

$$
n^{2}(\log n)^{\frac{1}{2}+\gamma} n^{-2}(\log n)^{-2 \gamma}+\frac{n^{-1}}{n^{-2}(\log n)^{-\frac{1}{2}-\gamma}} n^{\frac{1}{10}} n^{-2}(\log n)^{-2 \gamma}=O\left((\log n)^{\frac{1}{2}-\gamma}\right) .
$$

The factor $n^{\frac{1}{10}}$ arises because after a principal axis transformation the vector $T\left(x_{\alpha}\right)$, for all $x_{\alpha} \in \mathcal{B}$, will have standard deviation approximately one and $n^{-1 / 10}$ in the respective coordinate directions. We conclude that

$$
\lim _{n \rightarrow \infty} \mathbb{P}\left(\left\{\min _{x \in \mathbb{T}}|T(x)|<n^{-\frac{1}{2}}(\log n)^{-\gamma}\right\}\right)=0
$$

for standard normal $r_{j}$ and $\gamma>\frac{1}{2}$.

A proof of Theorem 1.2 along these lines has to overcome two main obstacles. First, in the case of Rademacher coefficients one cannot simply invoke the central limit theorem to estimate the sum over $\alpha$ in (2.2), since the error introduced by normal approximation can be as large as $n^{-\frac{1}{2}}$. Second, since the Salem-Zygmund inequality is sharp, we shall need to be more careful if we wish to avoid the loss of a logarithm - the main idea will be to consider the joint distribution of $\left(T, T^{\prime}\right)$. On the other hand, passing to general $\phi$ and extending the estimates to a neighborhood of the unit circle will turn out to be of a more technical nature. Returning to the first obstacle, note that the probabilities in the sum over $\alpha$ in (2.2) can be much larger than $n^{-2}$. For example, it is easy to see that $\mathbb{P}\left(\left\{T\left(\frac{\pi}{2}\right)=0\right\}\right) \sim \frac{1}{\pi n}$ if $n \rightarrow \infty$ through multiples of 4 . One cannot expect to obtain an $n^{-2}$-estimate in this case, since all values of $T\left(\frac{\pi}{2}\right)$ are in $\mathbb{Z}^{2}$. The same remark applies to other fractions $2 \pi \frac{h}{k}$ with small denominators, e.g., $\frac{\pi}{3}, \frac{2 \pi}{3}$. On the other hand, it will turn out that the desired estimate on the probabilities in (2.2) does hold for all $x_{\alpha}$ which do not come close to such fractions.

We begin with the proof of Theorem 1.2. Let $r_{j}$ and $\phi$ be as in the hypothesis of Theorem 1.2. We assume throughout that $\sigma \in(1 / 2,1 / 2+1 / 20)$ and that

Define

$$
\|\phi\|_{C^{\sigma}}=\max _{0 \leq t \leq 1}|\phi(t)|+\sup _{0 \leq t<s \leq 1} \frac{|\phi(t)-\phi(s)|}{|t-s|^{\sigma}}=1 .
$$

$$
T(x)=\sum_{j=0}^{n-1} r_{j} \phi(j / n) e^{i j x} \quad \text { and } \quad p(z)=\sum_{j=0}^{n-1} r_{j} \phi(j / n) z^{j} .
$$


Let

$$
\left\{y_{\beta}\right\}_{\beta=1}^{B}=\left\{2 \pi \frac{h}{k}: 1 \leq k \leq A, 0 \leq h \leq k-1,(h, k)=1\right\}
$$

where $A$, and thus $B$, are constants depending only on $\phi$. A will be specified below. Fix some $\epsilon>0$ (the same $\epsilon$ as in Theorem 1.2) and split $\mathbb{T}$ into non-overlapping intervals $I_{\alpha}$ of length between $\frac{1}{2} \epsilon n^{-2}$ and $\epsilon n^{-2}$.

Definition 2.1. The intervals $J_{\beta}=\left[y_{\beta}-2 \pi n^{-1+\sigma / 20}, y_{\beta}+2 \pi n^{-1+\sigma / 20}\right], \beta=$ $1,2, \ldots, B$, will be called bad. We define $I_{\alpha}$ to be good provided $I_{\alpha} \not \subset \bigcup_{\beta=1}^{B} J_{\beta}$. For any such $I_{\alpha}$ fix an $x_{\alpha} \in I_{\alpha} \backslash \bigcup_{\beta=1}^{B} J_{\beta}$. Furthermore, set

$$
\begin{aligned}
\mathcal{N} & =\left\{z \in \mathbb{C}:|| z|-1|<\epsilon n^{-2}\right\}, \\
\mathcal{G} & =\left\{\left\|T^{\prime}\right\|_{\infty} \leq C_{0} n^{\frac{3}{2}}(\log n)^{\frac{1}{2}}, \sup _{z \in \mathcal{N}}\left|p^{\prime \prime}(z)\right| \leq n^{13 / 4}\right\}
\end{aligned}
$$

where $C_{0}$ is a sufficiently large absolute constant.

For any interval $I \subset \mathbb{T}$ we denote $\mathcal{E}(I)=\left\{e^{i x}: x \in I\right\}$. By $I_{\alpha}$ we shall henceforth mean a good interval. Let $D\left(z_{0}, \rho\right)=\left\{z \in \mathbb{C}:\left|z-z_{0}\right|<\rho\right\}$. In analogy to (2.1) we now have

$$
\begin{aligned}
\mathbb{P}\left(\left\{\min _{z \in \mathcal{N}}|p(z)|<\epsilon n^{-\frac{1}{2}}\right\}\right) & \leq \sum_{\alpha} \mathbb{P}\left(\left\{\min _{z \in D\left(e^{\left.i x_{\alpha}, 2 \epsilon n^{-2}\right)}\right.}|p(z)|<\epsilon n^{-\frac{1}{2}}\right\} \cap \mathcal{G}\right) \\
& +\sum_{\beta=1}^{B} \mathbb{P}\left(\left\{\min _{z \in \mathcal{N}, z /|z| \in \mathcal{E}\left(J_{\beta}\right)}|p(z)|<\epsilon n^{-\frac{1}{2}}\right\} \cap \mathcal{G}\right)+\mathbb{P}\left(\mathcal{G}^{c}\right) .
\end{aligned}
$$

To avoid the loss of a logarithmic factor, we shall use Taylor polynomials of $T$ of order two around $e^{i x_{\alpha}}$ to estimate the sum over $\alpha$. If the event $\mathcal{G}$ occurs, then

$p(z)=T\left(x_{\alpha}\right)-\left(z-e^{i x_{\alpha}}\right) i e^{-i x_{\alpha}} T^{\prime}\left(x_{\alpha}\right)+O\left(\epsilon^{2} n^{-3 / 4}\right)$ for all $z \in D\left(e^{i x_{\alpha}}, 2 \epsilon n^{-2}\right)$.

Hence, if $|p(z)|<\epsilon n^{-\frac{1}{2}}$ for some $z \in D\left(e^{i x_{\alpha}}, 2 \epsilon n^{-2}\right)$, then

$$
\left|T\left(x_{\alpha}\right)-\left(z-e^{i x_{\alpha}}\right) i e^{-i x_{\alpha}} T^{\prime}\left(x_{\alpha}\right)\right|<2 \epsilon n^{-\frac{1}{2}}
$$

for large $n$. Consequently, if $\left|T\left(x_{\alpha}\right)\right| \geq 4 \epsilon n^{-2}\left|T^{\prime}\left(x_{\alpha}\right)\right|$ also, then $\left|T\left(x_{\alpha}\right)\right|<4 \epsilon n^{-\frac{1}{2}}$. We conclude that for each $I_{\alpha}$,

$$
\begin{aligned}
& \mathbb{P}\left(\left\{\min _{z \in D\left(e^{i x_{\alpha}}, 2 \epsilon n^{-2}\right)}|p(z)|<\epsilon n^{-\frac{1}{2}}\right\} \cap \mathcal{G}\right) \leq \mathbb{P}\left(\left\{\left|T\left(x_{\alpha}\right)\right|<4 \epsilon n^{-\frac{1}{2}}\right\}\right) \\
& \quad+\mathbb{P}\left(\left\{\left|T\left(x_{\alpha}\right)\right| \leq 4 \epsilon n^{-2}\left|T^{\prime}\left(x_{\alpha}\right)\right|,\left\|T^{\prime}\right\|_{\infty} \leq C_{0} n^{\frac{3}{2}}(\log n)^{\frac{1}{2}}\right\}\right) .
\end{aligned}
$$

In sections 4 and 5 we shall prove that the right-hand side of $(2.5)$ is $O\left(\epsilon^{2} n^{-2}\right)$ as $n \rightarrow \infty$. Since the number of good intervals does not exceed $4 \pi n^{2} \epsilon^{-1}$, this will imply that the sum over $\alpha$ in (2.4) is $O(\epsilon)$ as $n \rightarrow \infty$. In the following section we shall establish that the sum over the bad intervals in (2.4) is $o(1)$ as $n \rightarrow \infty$. Thus the proof of Theorem 1.2 will be complete provided $\lim _{n \rightarrow \infty} \mathbb{P}\left(\mathcal{G}^{c}\right)=0$. Clearly,

$$
\mathbb{P}\left(\mathcal{G}^{c}\right) \leq \mathbb{P}\left(\left\{\left\|T^{\prime}\right\|_{\infty}>C_{0} n^{\frac{3}{2}}(\log n)^{\frac{1}{2}}\right\}\right)+\mathbb{P}\left(\left\{\sup _{z \in \mathcal{N}}\left|p^{\prime \prime}(z)\right|>n^{13 / 4}\right\}\right) .
$$

The first term is $O\left(n^{-1}\right)$ by the Salem-Zygmund inequality provided $C_{0}$ is a sufficiently large absolute constant (see [3], chapter 6, Theorem 2). We estimate the 
second term using Markov's inequality.

$$
\begin{aligned}
\mathbb{P}\left(\left\{\sup _{z \in \mathcal{N}}\left|p^{\prime \prime}(z)\right|>n^{13 / 4}\right\}\right) & \leq \mathbb{P}\left(\left\{\sum_{j=0}^{n-1}\left|r_{j}\right||\phi(j / n)| j(j-1)\left(1+1 / n^{2}\right)^{j-2}>n^{13 / 4}\right\}\right) \\
& \leq n^{-13 / 4} \sum_{j=0}^{n-1} \mathbb{E}\left|r_{j}\right| j^{2} e \leq C n^{-1 / 4}
\end{aligned}
$$

\section{BAD INTERVALS}

We shall estimate the sum over the bad intervals in (2.4) using the two-dimensional version of the Berry-Esseen theorem for non-identically distributed random variables with bounded third moments (cf. Corollary 17.2 in [1]). To do so, we first need to bound the eigenvalues of the mean covariance matrix of $T\left(x_{\alpha}\right)$. We begin with a technical lemma that will be used repeatedly. In what follows, $C, c$ will denote large and small constants, respectively, depending only on $\phi$.

Lemma 3.1. For any $x \in(4 / n, \pi-4 / n)$ and nonnegative integer $\nu$

$$
\frac{1}{n}\left|\sum_{j=0}^{n-1} \phi(j / n)^{2}(j / n)^{\nu} e^{2 i j x}\right| \leq C(\nu+1) \min \left[(n \sin x)^{-\sigma}+\sin x,\left(n^{\sigma} \sin x\right)^{-1}\right] .
$$

In particular, with $x_{\alpha}$ as in Definition 2.1,

$$
\begin{aligned}
\frac{1}{n} \sum_{j=0}^{n-1} \phi(j / n)^{2}(j / n)^{\nu} e^{2 i j x_{\alpha}} & =o(1) \\
\frac{1}{n} \sum_{j=0}^{n-1} \phi(j / n)^{2}(j / n)^{\nu} \cos ^{2}\left(j x_{\alpha}\right) & =\frac{1}{2} \int_{0}^{1} \phi(t)^{2} t^{\nu} d t+o(1)
\end{aligned}
$$

uniformly in $\alpha$ as $n \rightarrow \infty$.

Proof. We first assume that $\nu=0$. That the left-hand side of (3.1) is bounded by $\left(n^{\sigma} \sin x\right)^{-1}$, follows by partial summation using that

$$
\sum_{j=0}^{k} e^{2 i j x}=\frac{\sin ((k+1) x)}{\sin x} e^{i k x} .
$$

For the other part of the estimate let $l=\left[\frac{\pi}{\sin x}\right]$. Note that $l<n$ by our choice of $x$. Then

$$
\sum_{j=k l}^{(k+1) l-1} e^{2 i j x}=\frac{\sin (l x)}{\sin x} e^{i((2 k+1) l-1) x}=O(1)
$$

and thus

$$
\begin{aligned}
\sum_{j=k l}^{(k+1) l-1} \phi(j / n)^{2} e^{2 i j x}= & \sum_{j=k l}^{(k+1) l-1} \phi(k l / n)^{2} e^{2 i j x} \\
& +\sum_{j=k l}^{(k+1) l-1}\left[\phi(j / n)^{2}-\phi(k l / n)^{2}\right] e^{2 i j x} \\
= & O(1)+l O\left((l / n)^{\sigma}\right) .
\end{aligned}
$$


Therefore one finally has

$$
\begin{aligned}
\frac{1}{n} \sum_{j=0}^{n-1} \phi(j / n)^{2} e^{2 i j x}= & \frac{1}{n} \sum_{k=0}^{[n / l]-1} \sum_{j=k l}^{(k+1) l-1} \phi(j / n)^{2} e^{2 i j x} \\
& +\frac{1}{n} \sum_{j=[n / l] l}^{n-1} \phi(j / n)^{2} e^{2 i j x} \\
= & O\left(\frac{1}{n} \frac{n}{l}+\frac{1}{n} \frac{n}{l} l(l / n)^{\sigma}\right)+O(l / n) \\
= & O\left((n \sin x)^{-\sigma}+\sin x\right),
\end{aligned}
$$

as claimed. To obtain the estimate for positive $\nu$ simply note that

$$
\left\|t^{\nu} \phi\right\|_{C^{\sigma}} \leq \nu+1
$$

The second statement follows easily from $\cos (2 x)=2 \cos ^{2}(x)-1$ and (3.1) since

$$
\left|x_{\alpha}\right| \in\left[2 \pi n^{-1+\sigma / 20}, \pi-2 \pi n^{-1+\sigma / 20}\right]
$$

by Definition 2.1 .

As noted in section 2, the mean covariance matrix of $T(x)$ becomes "increasingly singular" as $x \rightarrow 0$ or $x \rightarrow \pi$. This is to be expected since $T(0)$ and $T(\pi)$ are real. Lemma 3.2 is a quantitative formulation of this fact.

Lemma 3.2. Let $0<\lambda(x) \leq \Lambda(x)$ denote the eigenvalues of the mean covariance matrix of $T(x)$, i.e., $V(x)=\frac{1}{n} \sum_{j=0}^{n-1} \operatorname{cov}\left(r_{j} \phi(j / n)(\cos (j x), \sin (j x))\right)$. For $n$ sufficiently large there exist constants $C, c$ so that

1. in the range $n^{-1} \leq|x| \leq \pi-n^{-1}$,

$$
c \leq \lambda(x) \leq \Lambda(x) \leq C
$$

2. if $C n^{-1-\sigma / 2}<|x|<n^{-1}$ or $C n^{-1-\sigma / 2}<|x-\pi|<n^{-1}$, then

$$
c(n|x|)^{2} \leq \lambda(x) \leq C, c \leq \Lambda(x) \leq C .
$$

Proof. By definition

$$
V(x)=\frac{1}{n} \sum_{j=0}^{n-1} \phi(j / n)^{2}\left[\begin{array}{cc}
\cos ^{2}(j x) & \frac{1}{2} \sin (2 j x) \\
\frac{1}{2} \sin (2 j x) & \sin ^{2}(j x
\end{array}\right] .
$$

Let $I_{1}=\int_{0}^{1} \phi(t)^{2} d t$. On the one hand, by the previous lemma,

$$
V(x)=\frac{1}{2}\left[\begin{array}{cc}
I_{1} & 0 \\
0 & I_{1}
\end{array}\right]+O\left(\frac{1}{n^{\sigma}|\sin x|}\right)
$$

provided $4 / n<|x|<\pi-4 / n$. On the other hand,

$$
\begin{aligned}
V(x)= & {\left[\begin{array}{cc}
\int_{0}^{1} \phi(t)^{2} \cos ^{2}(\operatorname{tn} x) d t & \frac{1}{2} \int_{0}^{1} \phi(t)^{2} \sin (2 \operatorname{tn} x) d t \\
\frac{1}{2} \int_{0}^{1} \phi(t)^{2} \sin (2 \operatorname{tn} x) d t & \int_{0}^{1} \phi(t)^{2} \sin ^{2}(\operatorname{tn} x) d t
\end{array}\right] } \\
& +O\left(|x|+\frac{1}{n^{\sigma}}\right) .
\end{aligned}
$$


Simply note that the variation of each integrand over intervals of length $\frac{1}{n}$ is $O\left(|x|+\frac{1}{n^{\sigma}}\right)$. It follows from (3.4) that

$$
\begin{aligned}
4 \operatorname{det} V(x)+O\left(|x|+\frac{1}{n^{\sigma}}\right)= & I_{1}^{2}-\left(\int_{0}^{1} \phi(t)^{2} \cos (2 \operatorname{tn} x) d t\right)^{2} \\
& -\left(\int_{0}^{1} \phi(t)^{2} \sin (2 t n x) d t\right)^{2} \\
= & I_{1}^{2}-\left|\int_{0}^{1} \phi(t)^{2} e^{2 i t n x} d t\right|^{2} \\
= & I_{1}^{2}-\int_{0}^{1} \int_{0}^{1} \phi(t)^{2} \phi(s)^{2} \cos (2(t-s) n x) d t d s \\
= & 2 \int_{0}^{1} \int_{0}^{1} \phi(t)^{2} \phi(s)^{2} \sin ^{2}((t-s) n x) d t d s .
\end{aligned}
$$

Consequently, if $|n x| \leq \pi / 2$, we have $|\sin ((t-s) n x)| \geq \frac{2}{\pi}|t-s||n x|$ and thus

$$
\begin{aligned}
4 \operatorname{det} V(x) & \geq \frac{8}{\pi^{2}} \int_{0}^{1} \int_{0}^{1} \phi(t)^{2} \phi(s)^{2}|t-s|^{2} d t d s|n x|^{2}-O\left(|x|+\frac{1}{n^{\sigma}}\right) \\
& \geq c|n x|^{2}
\end{aligned}
$$

provided $|x| \geq C n^{-1-\sigma / 2}$. If $|n x| \geq \pi / 2$ consider the positive continuous function on $a>0$

$$
D(a)=\int_{0}^{1} \int_{0}^{1} \phi(t)^{2} \phi(s)^{2} \sin ^{2}((t-s) a) d t d s
$$

Since

$$
\int_{0}^{1} \phi(t) \sin (b t) d t \rightarrow 0, \quad \int_{0}^{1} \phi(t) \cos (b t) d t \rightarrow 0 \quad(b \rightarrow \infty),
$$

one has $D(a) \rightarrow \frac{1}{2} I_{1}^{2}$ as $a \rightarrow \infty$ and $D(a)>c$ on $1<a<\infty$. Thus

$$
\operatorname{det} V(x) \geq c \quad \text { for } \quad \frac{\pi}{2 n}<|x|<c .
$$

Since always trace $V=I_{1}+O\left(\frac{1}{n^{\sigma}}\right)$, the lemma follows from (3.3), (3.5), and (3.6) (note that it suffices to consider small $x$ since $T(x)$ and $T(x+\pi)$ are identically distributed).

In the following lemma we show that the sum over $\beta$ in (2.4) is negligible as $n \rightarrow \infty$. For the terminology see Definition 2.1.

Lemma 3.3. As $n \rightarrow \infty$

$$
\sup _{1 \leq \beta \leq B} \mathbb{P}\left(\left\{\min _{z \in \mathcal{N}, z /|z| \in \mathcal{E}\left(J_{\beta}\right)}|p(z)|<\epsilon n^{-\frac{1}{2}}\right\} \cap \mathcal{G}\right)=o(1) .
$$

Proof. We may assume that $J_{1}=\left\{|x| \leq 2 \pi n^{-1+\sigma / 20}\right\}$ and $J_{2}=\{|x-\pi| \leq$ $\left.2 \pi n^{-1+\sigma / 20}\right\}$. Note that all other bad intervals are inside $\left\{n^{-1} \leq|x| \leq \pi-n^{-1}\right\}$. For $3 \leq \beta \leq B$ cover $J_{\beta}$ by non-overlapping intervals $J_{k}^{(\beta)}$ of length $n^{-\frac{5}{4}}(\log n)^{-\frac{1}{2}}$, $1 \leq k \leq k_{0}=5 \pi n^{\frac{1}{4}+\sigma / 20}(\log n)^{\frac{1}{2}}$. Let $x_{k}^{(\beta)}$ denote the center of $J_{k}^{(\beta)}$. Suppose 
$|p(z)|<\epsilon n^{-\frac{1}{2}}$ for some $z \in \mathcal{N}, z /|z|=e^{i x} \in J_{k}^{(\beta)}$ and that the event $\mathcal{G}$ occurs. Expanding $p$ to second order around $e^{i x}$ one obtains

$$
|T(x)|=\left|p\left(e^{i x}\right)\right|<\epsilon n^{-\frac{1}{2}}+\epsilon n^{-2}\left|T^{\prime}(x)\right|+\epsilon^{2} n^{-4} n^{13 / 4}<2 C_{0} \epsilon n^{-\frac{1}{2}}(\log n)^{\frac{1}{2}} .
$$

Thus

$$
\left|T\left(x_{k}^{(\beta)}\right)\right| \leq|T(x)|+n^{-\frac{5}{4}}(\log n)^{-\frac{1}{2}}\left\|T^{\prime}\right\|_{\infty} \leq C n^{\frac{1}{4}} .
$$

It follows from the Berry-Essen theorem (see Corollary 17.2 in [1], and Lemma 3.2 above) that

$$
\mathbb{P}\left(\left\{\min _{z \in \mathcal{N}, z /|z| \in \mathcal{E}\left(J_{k}^{(\beta)}\right)}|p(z)|<\epsilon n^{-\frac{1}{2}}\right\} \cap \mathcal{G}\right) \leq \mathbb{P}\left(\left\{\left|T\left(x_{k}^{(\beta)}\right)\right| \leq C n^{\frac{1}{4}}\right\}\right) \leq C n^{-\frac{1}{2}}
$$

and thus

$$
\mathbb{P}\left(\left\{\min _{z \in \mathcal{N}, z /|z| \in \mathcal{E}\left(J_{\beta}\right)}|p(z)|<\epsilon n^{-\frac{1}{2}}\right\} \cap \mathcal{G}\right) \leq \sum_{k=1}^{k_{0}} C n^{-\frac{1}{2}} \leq C n^{-\frac{1}{4}+\sigma / 20}(\log n)^{\frac{1}{2}} .
$$

It remains to consider $J_{1} . J_{2}$ then follows by symmetry. Since the constant in the Berry-Essen theorem is proportional to the $(-3 / 2)-$ power of the smallest eigenvalue of the covariance matrix, Lemma 3.2 shows that we need to consider very small $x$ separately. Cover $\left\{n^{-1-\sigma / 16}<|x|<2 \pi n^{-1+\sigma / 20}\right\}$ by intervals $J_{k}^{(1)}$ of length $n^{-\frac{5}{4}}(\log n)^{-\frac{1}{2}}$ with center $x_{k}^{(1)}$. In view of (3.2), Corollary 17.2 in [1] implies

$$
\mathbb{P}\left(\left\{\left|T\left(x_{k}^{(1)}\right)\right|<C n^{\frac{1}{4}}\right\}\right) \leq C \lambda\left(x_{k}^{(1)}\right)^{-\frac{3}{2}} n^{-\frac{1}{2}} \leq C n^{3 \sigma / 16} n^{-\frac{1}{2}}
$$

whereas by the one-dimensional version of the Berry-Esseen theorem, see Theorem 12.4 in [1],

$$
\mathbb{P}\left(\left\{|T(0)| \leq C n^{1 / 2-\sigma / 16}(\log n)^{\frac{1}{2}}\right\}\right) \leq C n^{-\sigma / 16}(\log n)^{\frac{1}{2}} .
$$

Using Taylor expansions of second order as before we can therefore estimate

$$
\begin{aligned}
\mathbb{P}\left(\left\{\min _{z \in \mathcal{N}, z /|z| \in \mathcal{E}\left(J_{1}\right)}|p(z)|<\epsilon n^{-\frac{1}{2}}\right\} \cap \mathcal{G}\right) \leq & \sum_{k=1}^{k_{0}} \mathbb{P}\left(\left\{\left|T\left(x_{k}^{(1)}\right)\right| \leq C n^{\frac{1}{4}}\right\}\right) \\
& +\mathbb{P}\left(\left\{|T(0)|<C n^{1 / 2-\sigma / 16}(\log n)^{\frac{1}{2}}\right\}\right) \\
\leq & C n^{3 \sigma / 16+\sigma / 20-1 / 4}(\log n)^{\frac{1}{2}} \\
& +C n^{-\sigma / 16}(\log n)^{\frac{1}{2}}=o(1)
\end{aligned}
$$

as $n \rightarrow \infty$.

\section{Good intervals I}

In the following two sections we estimate the right-hand side of (2.5). We shall assume that $r_{0}, r_{1}, \ldots$ are Rademacher variables. As indicated in section 2 it is much easier to deal with standard normal variables since the vectors in (2.5) will again be Gaussian. The details are implicit in what follows. Moreover, in that case one does not need to introduce bad intervals because the issue of small denominators does not arise. The main result of this section is the following lemma.

Lemma 4.1. For any $\epsilon>0$ and sufficiently large $n$

$$
\sup _{\alpha} \mathbb{P}\left(\left\{\left|T\left(x_{\alpha}\right)\right|<\epsilon n^{-\frac{1}{2}}\right\}\right) \leq C \epsilon^{2} n^{-2} .
$$


Proof. The proof will use a method from [6] which allows us to handle very small probabilities. The idea is to approximate the characteristic functions of $T\left(x_{\alpha}\right)$ by Gaussians rather than the distribution functions. Generally speaking, this approximation will be possible only on a certain neighborhood of the origin. Outside of this neighborhood we shall show that the characteristic function is exponentially small. This property will depend crucially on the arithmetic properties of good intervals, which we shall exploit in the proof of Lemma 4.3 below.

Choose $\rho \in C^{\infty}\left(\mathbb{R}^{2}\right)$ such that $\rho \geq 0, \rho \geq 1$ on $D(0,1)$, and $\operatorname{supp}(\hat{\rho}) \subset D(0,2)$. Here and in what follows $\hat{\rho}$ denotes the Fourier transform

$$
\hat{\rho}(\xi)=\int_{\mathbb{R}^{2}} e^{-2 \pi i \xi \cdot x} \rho(x) d x .
$$

To see that $\rho$ exists, it suffices to take any $\eta \in C^{\infty}\left(\mathbb{R}^{2}\right) \backslash\{0\}$ with $\hat{\eta} \geq 0$ and $\operatorname{supp}(\hat{\eta}) \subset D(0,1)$ and to set $\rho(\cdot)=a|\eta|^{2}\left(b^{-1} \cdot\right)$. Here $a, b \geq 1$ are chosen so that $\rho \geq 1$ on $D(0,1)$. Note that $\operatorname{supp}(\hat{\rho})=\operatorname{supp}\left(a b^{2}(\hat{\eta} * \hat{\bar{\eta}})(b \cdot)\right) \subset D(0,2)$. Now let $\mathbb{P}_{\alpha}$ and $f_{\alpha}$ denote the distribution and the characteristic function of $\frac{1}{\sqrt{n}} T\left(x_{\alpha}\right)$, respectively. By Plancherel's theorem

$$
\begin{aligned}
\mathbb{P}\left(\left\{\left|T\left(x_{\alpha}\right)\right|<\epsilon n^{-\frac{1}{2}}\right\}\right) & \leq \int_{\mathbb{R}^{2}} \rho\left(\epsilon^{-1} n X\right) d \mathbb{P}_{\alpha}(X) \\
& =\epsilon^{2} n^{-2}\left\{\int_{I}+\int_{I I}\right\} \hat{\rho}\left(-\epsilon n^{-1} \xi\right) f_{\alpha}(\xi) d \xi
\end{aligned}
$$

where

$$
I=\left\{\xi \in \mathbb{R}^{2}:|\xi| \leq n^{\frac{1}{6}}\right\}, I I=\left\{\xi \in \mathbb{R}^{2}: n^{\frac{1}{6}}<|\xi| \leq 2 \epsilon^{-1} n\right\} .
$$

Note that the integrand in (4.1) vanishes for $|\xi|>2 \epsilon^{-1} n$. According to Definition 2.1, all good intervals $I_{\alpha}$ lie inside $\left\{2 \pi n^{-1+\sigma / 20}<|x|<\pi-2 \pi n^{-1+\sigma / 20}\right\}$. By Lemma 3.2 the covariance matrices $V_{\alpha}$ of $T\left(x_{\alpha}\right)$ are therefore uniformly nonsingular in $\alpha$ and $n$. Moreover, $T\left(x_{\alpha}\right)$ is the sum of independent random vectors of mean zero and uniformly bounded third moments. By Theorem 8.4 in [1],

$$
\left|f_{\alpha}(\xi)-\exp \left(-\left\langle V_{\alpha} \xi, \xi\right\rangle / 2\right)\right| \leq C|\xi|^{3} n^{-\frac{1}{2}} \exp \left(-c|\xi|^{2}\right)
$$

for all $|\xi| \leq n^{\frac{1}{6}}$. Here $C, c$ are absolute constants. Thus

$$
\int_{I} \hat{\rho}\left(-\epsilon n^{-1} \xi\right) f_{\alpha}(\xi) d \xi=\int_{I} \hat{\rho}\left(-\epsilon n^{-1} \xi\right) \exp \left(-\left\langle V_{\alpha} \xi, \xi\right\rangle / 2\right) d \xi+O\left(n^{-\frac{1}{2}}\right)
$$

is uniformly bounded in $\alpha$ and $n$. The boundedness of the integral over region $I I$ follows from Lemma 4.2 below.

In what follows $\tau$ will denote a fixed small constant, say $\tau=(\sigma-1 / 2) / 10$.

Lemma 4.2. For sufficiently large $n$ and all $\alpha$

$$
\sup _{n^{\frac{1}{6}}<|\xi|<n^{1+\tau}}\left|f_{\alpha}(\xi)\right|<\exp \left(-n^{\tau}\right) .
$$

Proof. Let $\xi=\left(\xi^{1}, \xi^{2}\right)$ be as in (4.2). Then $f_{\alpha}(\xi)=\prod_{j=0}^{n-1} \cos \left(\pi \psi_{j}\right)$ where

$$
\begin{aligned}
\psi_{j} & =\frac{1}{\pi} \frac{1}{\sqrt{n}} \phi(j / n)\left(\xi^{1} \cos \left(j x_{\alpha}\right)+\xi^{2} \sin \left(j x_{\alpha}\right)\right) \\
& =v \phi(j / n) \cos \left(j x_{\alpha}+\theta\right)
\end{aligned}
$$


for suitable $\theta$ and $v$ satisfying $\frac{1}{\pi} n^{-\frac{1}{3}}<v<n^{\frac{1}{2}+\tau}$. Since $\left|f_{\alpha}(\xi)\right|=1$ if all $\psi_{j} \in \mathbb{Z}$, we need to take into account how many $\psi_{j}$ are close to integers. Let ||$\cdot|| \mid$ denote the distance to the closest integer.

Case 1. $\operatorname{card}\left(\left\{j \in[0, n) \cap \mathbb{Z}:\left|\left\|\psi_{j} \mid\right\|>n^{-\tau}\right\}\right)>n^{4 \tau}\right.$. Then

$$
\begin{aligned}
\left|f_{\alpha}(\xi)\right| & =\exp \left(\sum_{j=0}^{n-1} \log \left(\cos \left(\pi \psi_{j}\right)\right)\right) \\
& \leq \exp \left(n^{4 \tau} \log \left(\cos \left(\pi n^{-\tau}\right)\right)\right) \leq \exp \left(-C n^{2 \tau}\right) .
\end{aligned}
$$

Case 2. $\operatorname{card}\left(\left\{j \in[0, n) \cap \mathbb{Z}:\left|\left\|\psi_{j}|\||>n^{-\tau}\right\}\right) \leq n^{4 \tau}\right.\right.$.

By Lemma 4.3 below there exists an interval $\mathcal{J} \subset[0, n)$ so that

$$
\sum_{j \in \mathcal{J}} \phi(j / n)^{2} \cos ^{2}\left(j x_{\alpha}+\theta\right)>c n^{1-5 \tau} \text { and } \sup _{j \in \mathcal{J}}\left|\psi_{j}\right| \leq 3 n^{-\tau}
$$

provided $n$ is large. In particular, $0<\cos \left(\pi \psi_{j}\right) \leq 1-\psi_{j}^{2}$ for all $j \in \mathcal{J}$. We therefore obtain from (4.3) and (4.5), uniformly in $\alpha$ and large $n$,

$$
\begin{aligned}
\sup _{n^{\frac{1}{6}}<|\xi|<n^{1+\tau}}\left|f_{\alpha}(\xi)\right| & \leq \sup _{\pi v>n^{-\frac{1}{3}}} \exp \left(-c \sum_{j \in \mathcal{J}} \psi_{j}^{2}\right) \leq \sup _{\pi v>n^{-\frac{1}{3}}} \exp \left(-c v^{2} n^{1-5 \tau}\right) \\
& \leq \exp \left(-c n^{\frac{1}{3}-5 \tau}\right)<\exp \left(-n^{\tau}\right) .
\end{aligned}
$$

For the last inequality note that $\tau \leq 1 / 20$.

The following lemma is the main technical statement of our paper. Roughly speaking, we show that if most of the $\psi_{j}$ given by (4.3) lie very close to integers, then many have to be close to zero (cf. (4.8)). This conclusion is false if $x_{\alpha}=\frac{\pi}{2}, \frac{\pi}{3}, \frac{\pi}{4}$ and $\theta=0$ and it is here that we therefore need to use that $x_{\alpha} / \pi$ is separated from fractions with small denominators. On the other hand, (4.7) is a simple consequence of Lemma 3.1.

Lemma 4.3. For any $j \in[0, n) \cap \mathbb{Z}$ let

$$
\psi_{j}=v \phi(j / n) \cos \left(j x_{\alpha}+\theta\right)
$$

where $x_{\alpha}$ is given by Definition 2.1, $v \in\left[0, n^{\frac{1}{2}+\tau}\right)$, and $\theta$ is arbitrary. Suppose that

$$
\operatorname{card}\left(\left\{j \in[0, n) \cap \mathbb{Z}:||\left|\psi_{j}\right|||>n^{-\tau}\right\}\right) \leq n^{4 \tau} .
$$

Then for large $n$ there exists an interval $\mathcal{J} \subset[0, n)$ so that

$$
\begin{aligned}
& \sum_{j \in \mathcal{J}} \phi(j / n)^{2} \cos ^{2}\left(j x_{\alpha}+\theta\right)>c n^{1-5 \tau}, \\
& \sup _{j \in \mathcal{J}}\left|\psi_{j}\right| \leq 3 n^{-\tau} .
\end{aligned}
$$

Proof. In the first part of the proof we select an interval $\mathcal{J} \subset[0, n)$ so that (4.7) holds and, moreover, $\left|\left\|\psi_{j} \mid\right\| \leq n^{-\tau}\right.$ for all $j \in \mathcal{J}$. For simplicity we shall write $x$ instead of $x_{\alpha}$.

Let $\mathcal{J}_{\nu}=\left[j_{\nu}, j_{\nu+1}\right)$ with $j_{\nu} \in \mathbb{Z}$ be disjoint intervals of length $\frac{1}{2} n^{1-5 \tau} \leq\left|\mathcal{J}_{\nu}\right| \leq$ $n^{1-5 \tau}$ such that

$$
[0, n)=\bigcup_{\nu=1}^{\nu_{0}} \mathcal{J}_{\nu} \quad \text { and } \quad \nu_{0} \leq 2 n^{5 \tau}
$$


Let

$$
s_{\nu}=\frac{1}{n} \sum_{j \in \mathcal{J}_{\nu}} \phi(j / n)^{2} \cos ^{2}(j x+\theta) .
$$

By Lemma 3.1 there exists a constant $c_{0}$ so that

$$
\sum_{\nu=1}^{\nu_{0}} s_{\nu}=\frac{1}{n} \sum_{j=0}^{n-1} \phi(j / n)^{2} \cos ^{2}(j x+\theta) \geq c_{0}^{2}
$$

provided $n$ is sufficiently large. We claim that

$$
s_{\nu}>\frac{1}{4} c_{0}^{2} n^{-5 \tau}
$$

for at least $2 n^{4 \tau}$ many choices of $\nu$. Suppose this fails. Since clearly $s_{\nu} \leq\left|\mathcal{J}_{\nu}\right| / n \leq$ $n^{-5 \tau}$ for all $\nu$, we would then have

$$
\sum_{\nu=1}^{\nu_{0}} s_{\nu} \leq \frac{1}{4} c_{0}^{2} n^{-5 \tau} \nu_{0}+2 n^{4 \tau} \cdot n^{-5 \tau} \leq \frac{1}{2} c_{0}^{2}+2 n^{-\tau}
$$

which contradicts (4.9) for large $n$. In view of hypothesis (4.6) we can therefore choose $\bar{\nu} \in\left\{1,2, \ldots, \nu_{0}\right\}$ so that

$$
\begin{aligned}
\sup _{j \in \mathcal{J}_{\bar{\nu}}}\left|\left\|\psi_{j} \mid\right\|\right. & \leq n^{-\tau}, \\
\sum_{j \in \mathcal{J}_{\bar{\nu}}} \phi(j / n)^{2} \cos ^{2}(j x+\theta) & >\frac{1}{4} c_{0}^{2} n^{1-5 \tau}=c_{1}^{2} n^{1-5 \tau} .
\end{aligned}
$$

We need to show that (4.8) holds for $\mathcal{J}=\mathcal{J}_{\bar{\nu}}$. The main idea of the proof will be that $\psi_{j}$ varies slowly as $j$ runs through certain arithmetic progressions. This can be seen by taking suitable differences of $\left\{\psi_{j}\right\}$. To do so, we first need to approximate $\phi$ by smooth functions. Extend $\phi$ to a $C^{\sigma}$-function on $\mathbb{T}$ with the same norm as follows. First extend $\phi$ to $[-1,1]$ as an even function and then set it constant equal to $\phi(1)$ on the remaining parts of $[-\pi, \pi]$. By Jackson's inequality (see [9], chapter III, Theorem 13.6) there exists a real trigonometric polynomial $Q$ of degree not exceeding $n A^{-2}$ such that

$$
\|\phi-Q\|_{\infty} \leq C_{0}\left(A^{2} n^{-1}\right)^{\sigma}
$$

with some absolute constant $C_{0}$. For all $j \in[0, n) \cap \mathbb{Z}$ let

$$
\tilde{\psi}_{j}=v Q(j / n) \cos (j x+\theta) .
$$

Since $v<n^{1 / 2+\tau}$ and $\tau=(\sigma-1 / 2) / 10,(4.10)$ implies that there exist integers $m_{j}$ for $j \in \mathcal{J}$ so that

$$
\sup _{j \in \mathcal{J}}\left[\left|\psi_{j}-m_{j}\right|+\left|\tilde{\psi}_{j}-m_{j}\right|\right] \leq 3 n^{-\tau} .
$$

We shall now show that $m_{j}$ is periodic with period $p_{0}$ satisfying $1 \leq p_{0} \leq A$. The constant $A$ is the same as in Definition 2.1 and will be specified below.

By Dirichlet's principle (see [2], section 11.3), there exist an integer $p_{0}$ and a real number $z_{0}$ so that

$$
p_{0} \frac{x}{2 \pi}-z_{0} \in \mathbb{Z}, \quad 1 \leq p_{0} \leq A, \quad\left|z_{0}\right| \leq A^{-1} .
$$


For any nonnegative integer $k$ define the $k^{t h}$ difference with respect to $l$ as usual, i.e.,

$$
\Delta^{k} \tilde{\psi}_{j+l p_{0}}=\sum_{s=0}^{k}\left(\begin{array}{l}
k \\
s
\end{array}\right)(-1)^{s} \tilde{\psi}_{j+(s+l) p_{0}} .
$$

To estimate this difference note first that for $u \in \mathbb{Z}$

$$
\tilde{\psi}_{j+u p_{0}}=v Q\left(\frac{j+u p_{0}}{n}\right) \cos \left(j x+\theta+2 \pi u z_{0}\right)
$$

and second that Bernstein's inequality (see [9], chapter X, Theorem 3.13) implies

$$
\left\|Q^{(s)}\right\|_{\infty} \leq\|Q\|_{\infty}\left(\frac{n}{A^{2}}\right)^{s}
$$

for any nonnegative integer $s$. Furthermore, $\|Q\|_{\infty} \leq 2$ for large $n$ in view of (4.12). Consequently, we can estimate (4.15) for any integers $l, k$ with $0 \leq j<p_{0}$ and $(k+l) p_{0}<n-j$ as follows:

$$
\begin{aligned}
\left|\Delta^{k} \tilde{\psi}_{j+l p_{0}}\right| & \leq\left\|\frac{d^{k}}{d u^{k}}\left[v Q\left(\frac{j+u p_{0}}{n}\right) \cos \left(j x+\theta+2 \pi u z_{0}\right)\right]\right\|_{L^{\infty}(u)} \\
& \leq v \sum_{s=0}^{k}\left(\begin{array}{c}
k \\
s
\end{array}\right)\left\|\frac{d^{s}}{d u^{s}} Q\left(\frac{j+u p_{0}}{n}\right)\right\|_{\infty}\left\|\frac{d^{k-s}}{d u^{k-s}} \cos \left(j x+\theta+2 \pi u z_{0}\right)\right\|_{\infty} \\
& \leq 2 v \sum_{s=0}^{k}\left(\begin{array}{c}
k \\
s
\end{array}\right)\left(\frac{n}{A^{2}}\right)^{s}\left(\frac{A}{n}\right)^{s}\left(2 \pi\left|z_{0}\right|\right)^{k-s} \leq 2 n^{\frac{1}{2}+\tau}\left(\frac{2 \pi+1}{A}\right)^{k} .
\end{aligned}
$$

In view of (4.13) and the definition of $\Delta^{k}(\mathrm{cf} .(4.15))$ we therefore have

$$
\begin{aligned}
\left|\Delta^{k} m_{j+l p_{0}}\right| & \leq\left|\Delta^{k} \tilde{\psi}_{j+l p_{0}}\right|+\left|\Delta^{k}\left(\tilde{\psi}_{j+l p_{0}}-m_{j+l p_{0}}\right)\right| \\
& \leq 2 n^{\frac{1}{2}+\tau}\left(\frac{2 \pi+1}{A}\right)^{k}+2^{k} \cdot 3 n^{-\tau}
\end{aligned}
$$

provided $\left[j+l p_{0}, j+(l+k) p_{0}\right] \subset \mathcal{J}$. Clearly, the expression on the right-hand side is $<1$ provided $k=[\tau \log n]$ and $A$ is chosen sufficiently large. Thus

$$
\Delta^{k} m_{j+l p_{0}}=0 \text { for all } j, l \in \mathbb{Z} \text { with }\left[j+l p_{0}, j+(l+k) p_{0}\right] \subset \mathcal{J} \text {. }
$$

In other words, $m_{j+l p_{0}}=P_{j}(l)$ for those $j$ and $l$, where $P_{j}$ is a real polynomial of degree $\leq k-1$. We claim that $P_{j}=$ const. Suppose not. Then as a nonzero polynomial of degree $<k-1, P_{j}^{\prime}$ has at most $k-2$ roots. In particular, $P_{j}$ has to be strictly increasing on an interval $\mathcal{J}^{\prime}$ of length $>|\mathcal{J}| /\left(k p_{0}\right)$. However, since $P_{j}$ attains one of the values $m_{0}, m_{1}, \ldots, m_{n-1} \in\left[-2 n^{\frac{1}{2}+\tau}, 2 n^{\frac{1}{2}+\tau}\right]$ at each integer in $\mathcal{J}^{\prime}$, and since $\left|\mathcal{J}^{\prime}\right| \geq n^{1-5 \tau} /\left(2 k p_{0}\right) \geq 5 n^{\frac{1}{2}+\tau}$, we obtain a contradiction to strict monotonicity. Thus

$$
m_{j+l p_{0}}=m_{j} \quad \text { for all } \quad j, l \in \mathbb{Z} \text { with }\left[j+l p_{0}, j+(l+k) p_{0}\right] \subset \mathcal{J} .
$$

Our next goal is to show that $m_{j}=0$ on $\mathcal{J}$. This will complete the proof (see (4.13)). We first consider the case

$$
\left|z_{0}\right|<n^{-1+\sigma / 20}
$$

Then by (4.14)

$$
x=x_{\alpha} \in\left[2 \pi \frac{q}{p_{0}}-2 \pi n^{-1+\sigma / 20}, 2 \pi \frac{q}{p_{0}}+2 \pi n^{-1+\sigma / 20}\right]
$$


for some integer $q$. This contradicts our choice of $x_{\alpha}$ (see Definition 2.1). Hence we may assume that

$$
\left|z_{0}\right| \geq n^{-1+\sigma / 20} \text {. }
$$

It is easy to see that for such $z_{0}$ the angles $\psi_{j}$ have to change sign on $\mathcal{J}$. This implies that $m_{j}=0$ by (4.13). We first show that $\phi(j / n)$ does not change sign on $\mathcal{J}$. Since $|\mathcal{J}| \leq n^{1-5 \tau},(4.11)$ implies

$$
\sup _{j \in \mathcal{J}} \phi(j / n)^{2} \geq \frac{1}{|\mathcal{J}|} \sum_{j \in \mathcal{J}} \phi(j / n)^{2} \cos ^{2}(j x+\theta)>c_{1}^{2} .
$$

Thus there exists $j_{0} \in \mathcal{J}$ so that w.l.o.g.

$$
\phi\left(j_{0} / n\right) \geq c_{1}
$$

Hence, for any $j \in \mathcal{J}$,

$$
\phi(j / n) \geq c_{1}-\left|\phi(j / n)-\phi\left(j_{0} / n\right)\right| \geq c_{1}-n^{-5 \sigma \tau}
$$

and so $\phi(j / n)>0$ on $\mathcal{J}$. By $(4.14)$

$$
\psi_{j+l p_{0}}=v \phi\left(\left(j+l p_{0}\right) / n\right) \cos \left(j x+\theta+2 \pi l z_{0}\right) .
$$

Recalling that $\sigma \in(1 / 2,1 / 2+1 / 20)$ and $\tau=(\sigma-1 / 2) / 10$ we see that (4.17) implies for large $n$

$$
|\mathcal{J}| \cdot\left|z_{0}\right| \geq \frac{1}{2} n^{1-5 \tau} n^{-1+\sigma / 20}>2 A .
$$

We can therefore find integers $l, l^{\prime}$ with $j+l p_{0}, j+l^{\prime} p_{0} \in \mathcal{J}$ satisfying

$$
\psi_{j+l p_{0}} \leq 0 \text { and } \quad \psi_{j+l^{\prime} p_{0}} \geq 0 .
$$

On the other hand, by (4.13) and (4.16),

$$
\left|\psi_{j+l p_{0}}-m_{j}\right|+\left|\psi_{j+l^{\prime} p_{0}}-m_{j}\right| \leq 6 n^{-\tau}
$$

In view of (4.18) this implies that $m_{j}=0$, and we are done.

\section{Good intervals II}

In Lemma 5.2 below we estimate the second term on the right-hand side of (2.5). By the discussion in section 2 this will complete the proof of Theorem 1.2. Let

$$
\begin{aligned}
X_{\alpha} & =\frac{1}{\sqrt{n}}\left(T\left(x_{\alpha}\right), T^{\prime}\left(x_{\alpha}\right) / i n\right), \\
\Omega & =\left\{(z, w) \in \mathbb{C}^{2}:|w| \leq C_{0} \sqrt{\log n},|z| \leq n^{-1} \epsilon|w|\right\} .
\end{aligned}
$$

Clearly,

$$
\mathbb{P}\left(\left\{\left|T\left(x_{\alpha}\right)\right| \leq \epsilon n^{-2}\left|T^{\prime}\left(x_{\alpha}\right)\right|,\left\|T^{\prime}\right\|_{\infty} \leq C_{0} n^{\frac{3}{2}}(\log n)^{\frac{1}{2}}\right\}\right) \leq \mathbb{P}\left(\left\{X_{\alpha} \in \Omega\right\}\right) .
$$

As in the previous section, we shall use an idea from [6] to bound this probability by $\epsilon^{2} n^{-2}$. First we need to consider the covariance matrix $W_{\alpha}$ of $X_{\alpha}$.

Lemma 5.1. Let

$$
W_{\alpha}=\frac{1}{n} \sum_{j=0}^{n-1} \operatorname{cov}\left(r_{j} \phi(j / n)\left(\cos \left(j x_{\alpha}\right), \sin \left(j x_{\alpha}\right), \frac{j}{n} \cos \left(j x_{\alpha}\right), \frac{j}{n} \sin \left(j x_{\alpha}\right)\right)\right)
$$

be the covariance matrix of $X_{\alpha}$. Then $W_{\alpha}$ is uniformly nonsingular in $\alpha$ and large $n$. 
Proof. Clearly, each entry in the matrix $W_{\alpha}$ is equal to one of the expressions

$$
\begin{aligned}
& \sum_{j=0}^{n-1} \phi(j / n)^{2}(j / n)^{\nu} \cos ^{2}\left(j x_{\alpha}\right), \sum_{j=0}^{n-1} \phi(j / n)^{2}(j / n)^{\nu} \sin ^{2}\left(j x_{\alpha}\right), \\
& \frac{1}{2} \sum_{j=0}^{n-1} \phi(j / n)^{2}(j / n)^{\nu} \sin \left(2 j x_{\alpha}\right)
\end{aligned}
$$

for some $\nu=0,1,2$. Therefore, Lemma 3.1 implies that as $n \rightarrow \infty$

$$
W_{\alpha}=\frac{1}{2}\left[\begin{array}{cccc}
I_{1} & 0 & I_{2} & 0 \\
0 & I_{1} & 0 & I_{2} \\
I_{2} & 0 & I_{3} & 0 \\
0 & I_{2} & 0 & I_{3}
\end{array}\right]+o(1)
$$

where $I_{1}=\int_{0}^{1} \phi(t)^{2} d t, I_{2}=\int_{0}^{1} t \phi(t)^{2} d t, I_{3}=\int_{0}^{1} t^{2} \phi(t)^{2} d t$. Note that the determinant of the matrix in (5.1) is $\frac{1}{16}\left(I_{1} I_{3}-I_{2}^{2}\right)^{2}>0$ since $I_{1} I_{3}-I_{2}^{2}$ is the Gram determinant composed of the linearly independent functions $\phi$ and $t \phi$ considered as elements of $L^{2}[0,1]$.

The following lemma is the main result of this section. As in the previous section, estimate (5.2) will be obtained by approximating the characteristic function of $X_{\alpha}$ on a certain neighborhood of the origin. Outside of this neighborhood we shall show that the characteristic function of $X_{\alpha}$ is exponentially small. This will again depend crucially on the arithmetic properties of good intervals, more precisely Lemma 4.3 above.

Lemma 5.2. For any $\epsilon>0$ and large $n$

$$
\sup _{\alpha} \mathbb{P}\left(\left\{X_{\alpha} \in \Omega\right\}\right) \leq C n^{-2} \epsilon^{2} .
$$

Proof. Let

$$
\begin{aligned}
& R_{0}=\left\{(z, w) \in \mathbb{C}^{2}:|w| \leq 1,|z| \leq n^{-1} \epsilon\right\} \quad \text { and } \\
& R_{j}=\left\{(z, w) \in \mathbb{C}^{2}: 2^{j-1} \leq|w| \leq 2^{j},|z| \leq 2^{j} n^{-1} \epsilon\right\} \quad \text { if } j \geq 1 .
\end{aligned}
$$

Clearly,

$$
\Omega \subset \bigcup_{j=0}^{j_{0}} R_{j}
$$

where $j_{0}$ is minimal with $2^{j_{0}-1}>C_{0}(\log n)^{\frac{1}{2}}$. Choose a nonnegative function $\chi \in C_{0}^{\infty}\left(\mathbb{R}^{2}\right)$ such that $\chi=0$ on a neighborhood of zero and $\chi \geq 1$ on $\{1 \leq|w| \leq 2\}$. Let $\rho$ be defined as in Lemma 4.1. Set

$$
F_{0}(z, w)=\rho(w) \rho\left(n \epsilon^{-1} z\right), \quad F_{j}(z, w)=\chi\left(2^{-j+1} w\right) \rho\left(2^{-j} n \epsilon^{-1} z\right) \quad \text { if } j \geq 1 .
$$

By (5.3) and the choice of $\chi$ and $\rho$,

$$
\sum_{j=0}^{j_{0}} F_{j} \geq \chi_{\Omega} .
$$


Let $\mathbb{P}_{X_{\alpha}}$ and $g_{\alpha}$ denote the distribution and characteristic function of $X_{\alpha}$, respectively. Then by Plancherel's theorem

$$
\begin{aligned}
\mathbb{P}\left(\left\{X_{\alpha} \in \Omega\right\}\right) \leq & \sum_{j=0}^{j_{0}} \int_{\mathbb{R}^{4}} F_{j}(z, w) d \mathbb{P}_{X_{\alpha}}(z, w) \\
= & n^{-2} \epsilon^{2} \int_{\mathbb{R}^{4}} \hat{\rho}(-\eta) \hat{\rho}\left(n^{-1} \epsilon \xi\right) g_{\alpha}(\xi, \eta) d \xi d \eta \\
& +\sum_{j=1}^{j_{0}} n^{-2} \epsilon^{2} 2^{4 j-2} \int_{\mathbb{R}^{4}} \hat{\chi}\left(-2^{j-1} \eta\right) \hat{\rho}\left(-2^{j} n^{-1} \epsilon \xi\right) g_{\alpha}(\xi, \eta) d \xi d \eta .
\end{aligned}
$$

It will suffice to estimate the sum over $j$. The reader will easily verify that the first integral (corresponding to $j=0$ ) in (5.4) can be dealt with in the same manner.

Split $\mathbb{R}^{4}$ into the regions (with $\tau=(\sigma-1 / 2) / 10$ as in section 4 )

$$
\begin{aligned}
I & =\left\{|\xi| \leq n^{\frac{1}{6}}, \quad|\eta| \leq n^{\tau}\right\}, \quad I I=\left\{n^{\frac{1}{6}}<|\xi| \leq n^{1+\tau}, \quad|\eta| \leq n^{\tau}\right\}, \\
I I I & =\left\{|\eta|>n^{\tau}\right\} .
\end{aligned}
$$

Note that the integrands in (5.4) vanish if $|\xi| \geq n^{1+\tau}$ and $\epsilon n^{\tau} \geq 2$. We shall assume this last condition. Since $\hat{\chi}$ is a Schwartz function, the integral over region $I I I$ will decay rapidly with increasing $j$. Indeed, denoting the integrand in the summands of (5.4) by $G_{j}$ and using $\left\|g_{\alpha}\right\|_{\infty} \leq 1$, we obtain

$$
\begin{aligned}
& n^{-2} \epsilon^{2} 2^{4 j-2}\left|\int_{I I I} G_{j}(\xi, \eta) d \xi d \eta\right| \\
\leq & n^{-2} \epsilon^{2} 2^{4 j-2} \int_{|\eta|>n^{\tau}} \int_{\mathbb{R}^{2}}\left|\hat{\chi}\left(-2^{j-1} \eta\right)\right|\left|\hat{\rho}\left(-2^{j} n^{-1} \epsilon \xi\right)\right| d \xi d \eta \\
\leq & C_{N} \int_{|\eta|>2^{j-1} n^{\tau}}(1+|\eta|)^{-2 N} d \eta \leq C_{N} 2^{-N j} n^{-N \tau}
\end{aligned}
$$

for any $N>1$. In particular, we can take $N \geq 3 \tau^{-1}$ so that

$$
\sum_{j=1}^{\infty} n^{-2} \epsilon^{2} 2^{4 j-2}\left|\int_{I I I} G_{j}(\xi, \eta) d \xi d \eta\right| \leq C n^{-3} .
$$

Next we turn to region I. Since $\left(T\left(x_{\alpha}\right), T^{\prime}\left(x_{\alpha}\right) /\right.$ in $)$ is the sum of independent random vectors with mean zero and uniformly bounded third moments Lemma 5.1 above and Theorem 8.4 in [1] imply

$$
\left|g_{\alpha}(\xi, \eta)-\exp \left(-\left|W_{\alpha}^{\frac{1}{2}}(\xi, \eta)\right|^{2} / 2\right)\right| \leq C n^{-\frac{1}{2}}(|\xi|+|\eta|)^{3} \exp \left(-c\left(|\xi|^{2}+|\eta|^{2}\right)\right)
$$

provided $|\xi|+|\eta| \leq n^{\frac{1}{6}}$. Hence

$$
\begin{aligned}
& \int_{I} \hat{\chi}\left(-2^{j-1} \eta\right) \hat{\rho}\left(-2^{j} n^{-1} \epsilon \xi\right) g_{\alpha}(\xi, \eta) d \xi d \eta \\
= & \int_{\mathbb{R}^{4}} \hat{\chi}\left(-2^{j-1} \eta\right) \hat{\rho}\left(-2^{j} n^{-1} \epsilon \xi\right) \exp \left(-\left|W_{\alpha}^{\frac{1}{2}}(\xi, \eta)\right|^{2} / 2\right) d \xi d \eta+O\left(n^{-\frac{1}{2}}\right) .
\end{aligned}
$$

Note that all moments of $\hat{\chi}$ vanish since

$$
\int_{\mathbb{R}^{2}} \hat{\chi}(\eta) \eta^{\gamma} d \eta=\left(2 \pi i \frac{\partial}{\partial z}\right)^{\gamma} \chi(0)=0
$$


In order to exploit this fact, we will approximate the Gaussian in (5.6) by a Taylor polynomial in $\eta$ for fixed $\xi$. This type of argument is standard in Littlewood-Paley theory from harmonic analysis. Let

$$
P_{m, \alpha}(\xi, \eta)=\left.\sum_{|\beta| \leq m} \frac{\eta^{\beta}}{\beta !} \frac{\partial^{\beta}}{\partial \zeta^{\beta}} \exp \left(-\left|W_{\alpha}^{\frac{1}{2}}(\xi, \zeta)\right|^{2} / 2\right)\right|_{\zeta=0} .
$$

Since $P_{m, \alpha}(\xi, \eta)$ is a polynomial in $\eta$ for fixed $\xi$, (5.7) implies that

$$
\int_{\mathbb{R}^{2}} \hat{\chi}(\eta) P_{m, \alpha}(\xi, \eta) d \eta=0
$$

for every $\xi \in \mathbb{R}^{2}$. Furthermore, it is easy to verify that

$$
\begin{aligned}
& \left|P_{m, \alpha}(\xi, \eta)\right| \leq C_{m}(1+|\xi|+|\eta|)^{m} \exp \left(-c|\xi|^{2}\right) \\
& \left|P_{m, \alpha}(\xi, \eta)-\exp \left(-\left|W_{\alpha}^{\frac{1}{2}}(\xi, \eta)\right|^{2} / 2\right)\right| \\
& \leq C_{m}(1+|\xi|+|\eta|)^{m+1} \cdot|\eta|^{m+1} \exp \left(-c|\xi|^{2}\right)
\end{aligned}
$$

Indeed, (5.10) follows from the definition (5.8) and the chain rule, whereas (5.11) is a consequence of the standard error estimates for the Taylor expansion. In view of (5.9)-(5.11) the integral in (5.6) can now be estimated as follows.

$$
\begin{aligned}
& \left|\int_{\mathbb{R}^{4}} \hat{\chi}\left(-2^{j-1} \eta\right) \hat{\rho}\left(-2^{j} n^{-1} \epsilon \xi\right) \exp \left(-\left|W_{\alpha}^{\frac{1}{2}}(\xi, \eta)\right|^{2} / 2\right) d \xi d \eta\right| \\
= & \left|\int_{\mathbb{R}^{4}} \hat{\chi}\left(-2^{j-1} \eta\right) \hat{\rho}\left(-2^{j} n^{-1} \epsilon \xi\right)\left[\exp \left(-\left|W_{\alpha}^{\frac{1}{2}}(\xi, \eta)\right|^{2} / 2\right)-P_{m, \alpha}(\xi, \eta)\right] d \xi d \eta\right| \\
\leq & C \int_{|\eta| \geq 1} \int_{\mathbb{R}^{2}}\left|\hat{\chi}\left(-2^{j-1} \eta\right)\right|\left[\left|P_{m, \alpha}(\xi, \eta)\right|+\exp \left(-\left|W_{\alpha}^{\frac{1}{2}}(\xi, \eta)\right|^{2} / 2\right)\right] d \xi d \eta \\
& +C \int_{|\eta| \leq 1} \int_{\mathbb{R}^{2}}\left|\hat{\chi}\left(-2^{j-1} \eta\right)\right|\left|P_{m, \alpha}(\xi, \eta)-\exp \left(-\left|W_{\alpha}^{\frac{1}{2}}(\xi, \eta)\right|^{2} / 2\right)\right| d \xi d \eta \\
\leq & C_{N, m} \int_{|\eta| \geq 1} \int_{\mathbb{R}^{2}}\left(2^{j}|\eta|\right)^{-N}\left[(1+|\xi|+|\eta|)^{m} \exp \left(-c|\xi|^{2}\right)\right. \\
& +C_{N, m} \int_{|\eta| \leq 1} \int_{\mathbb{R}^{2}}\left(1+2^{j}|\eta|\right)^{-N-1}(1+|\xi|)^{m+1}|\eta|^{m+1} \exp \left(-c|\xi|^{2}\right) d \xi d \eta \\
\leq & C_{N, m}\left(2^{-j N}+2^{-j(m+3)}\right)
\end{aligned}
$$

provided $N>m+2$. With $m=2, N=5$ we therefore conclude from (5.6) and (5.12) that

$$
\begin{aligned}
& \sum_{j=1}^{j_{0}} n^{-2} \epsilon^{2} 2^{4 j-2}\left|\int_{I} G_{j}(\xi, \eta) d \xi d \eta\right| \\
\leq & C \sum_{j=0}^{\infty} n^{-2} \epsilon^{2} 2^{4 j-2} 2^{-5 j}+C \sum_{j=0}^{j_{0}} n^{-2} \epsilon^{2} 2^{4 j-2} n^{-\frac{1}{2}} \leq C n^{-2} \epsilon^{2}
\end{aligned}
$$

for large $n$. Finally, Lemma 5.3 below implies that the contributions from region II are negligible. In view of (5.5) and (5.13) the proof is complete. 
Lemma 5.3. For all $\alpha$ and large $n$

$$
\sup _{(\xi, \eta) \in I I}\left|g_{\alpha}(\xi, \eta)\right|<\exp \left(-n^{\tau}\right) .
$$

Proof. The proof is very similar to that of Lemma 4.2. Let $(\xi, \eta) \in I I$. Then

$$
g_{\alpha}(\xi, \eta)=\prod_{j=0}^{n-1} \cos \left(\pi \beta_{j}\right)
$$

with

$$
\beta_{j}=v \phi(j / n) \cos \left(j x_{\alpha}+\theta_{0}\right)+w \frac{j}{n} \phi(j / n) \cos \left(j x_{\alpha}+\theta_{1}\right),
$$

for suitable $\theta_{0}, \theta_{1}$ and $v, w$ satisfying

$$
\frac{1}{\pi} n^{-\frac{1}{3}}<v<n^{\frac{1}{2}+\tau}, 0 \leq w \leq n^{-\frac{1}{2}+\tau} .
$$

By an argument analogous to (4.4) it suffices to assume that

$$
\operatorname{card}\left(\left\{j \in[0, n) \cap \mathbb{Z}:\left|\left\|\beta_{j} \mid\right\|>\frac{1}{2} n^{-\tau}\right\}\right) \leq n^{4 \tau} .\right.
$$

Define

$$
\psi_{j}=v \phi(j / n) \cos \left(j x_{\alpha}+\theta_{0}\right) .
$$

Since $0 \leq w \leq n^{-\frac{1}{2}+\tau}$ it follows from (5.15) that for large $n$

$$
\operatorname{card}\left(\left\{j \in[0, n) \cap \mathbb{Z}:||\left|\psi_{j}\right| \|>n^{-\tau}\right\}\right) \leq n^{4 \tau} .
$$

By Lemma 4.3 there exists an interval $\mathcal{J} \subset[0, n)$ so that for some constant $c$

$$
\sum_{j \in \mathcal{J}} \phi(j / n)^{2} \cos ^{2}\left(j x_{\alpha}+\theta_{0}\right) \geq c n^{1-5 \tau}
$$

and $\sup _{j \in \mathcal{J}}\left|\psi_{j}\right| \leq 3 n^{-\tau}$. This clearly implies that $\sup _{j \in \mathcal{J}}\left|\beta_{j}\right|<1 / 4$. Hence

$$
0<\cos \left(\pi \beta_{j}\right) \leq 1-\beta_{j}^{2}
$$

and thus

$$
\sup _{I I}\left|g_{\alpha}(\xi, \eta)\right| \leq \sup _{\pi v>n^{-1 / 3}, w \leq n^{-1 / 2+\tau}} \exp \left(-c \sum_{j \in \mathcal{J}} \beta_{j}^{2}\right) .
$$

It follows easily from (5.14) that

$$
\beta_{j}^{2} \geq \frac{v^{2}}{2} \phi(j / n)^{2} \cos ^{2}\left(j x_{\alpha}+\theta\right)-w^{2} \phi(j / n)^{2} .
$$

We therefore obtain in view of (5.16) and because of $v>\frac{1}{\pi} n^{-\frac{1}{3}}, 0 \leq w<n^{-\frac{1}{2}+\tau}$ that

$$
\sum_{j \in \mathcal{J}} \beta_{j}^{2} \geq c n^{\frac{1}{3}-5 \tau}-n^{-1+2 \tau} n \geq c n^{\frac{1}{3}-5 \tau}
$$

for large $n$, and the lemma follows (recall $\tau \leq 1 / 200$ ). 


\section{REFERENCES}

1. R. N. Bhattacharya, R. Rao. Normal approximation and asymptotic expansions. John Wiley \& Sons, New York, 1976. MR 55:9219

2. G. H. Hardy, E. M. Wright. An introduction to the theory of numbers. Fifth edition, Oxford University Press, Oxford, 1979. MR 81i:10002

3. J-P. Kahane. Some random series of functions. Second Edition, Cambridge University Press, Cambridge, 1985. MR 87m:60119

4. A. G. Karapetian. On the minimum of the absolute value of trigonometric polynomials with random coefficients. (Russian) Mat. Zametki 61 (1997), no. 3, 451-455. CMP 98:12

5. B. S. Kashin. The properties of random polynomials with coefficients \pm 1 . Vestn. Mosk. Univ. Ser. Mat.-Mekh., No. 5 (1987), 40-46. MR 89a:60135

6. S. V. Konyagin. On the minimum modulus of random trigonometric polynomials with coefficients \pm 1 . (Russian, Translation in Math. Notes) Mat. Zametki 56 (1994), no. 3, 80-101. MR 95k:42015

7. J. E. Littlewood. On polynomials $\sum^{n} \pm z^{m}, \sum^{n} e^{\alpha_{m} i} z^{m}, z=e^{\theta i}$. J. London Math. Soc. 41 (1966), 367-376. MR 33:4237

8. L. A. Shepp, R. J. Vanderbei. The complex zeros of random polynomials. Trans. Amer. Math. Soc. 347 (1995), no. 11, 4365-4384. MR 96a:30006

9. A. Zygmund. Trigonometric Series. Second Edition, Cambridge University Press, Cambridge, 1959. MR 21:6498

Institute for Advanced Study, School of Mathematics, Olden Lane, Princeton, New JERSEY 08540

Current address: Department of Mechanics and Mathematics, Moscow State University, Moscow, 119899, Russia

E-mail address: kon@nw.math.msu.su

Institute for Advanced Study, School of Mathematics, Olden Lane, Princeton, New JERSEY 08540

Current address: Department of Mathematics, Princeton University, Fine Hall, Princeton, New Jersey 08544

E-mail address: schlag@math.princeton.edu 